\title{
Adsorption Characteristics of Cadmium and Lead Heavy Metals into Locally Synthesized Chitosan Biopolymer
}

\author{
J.M. Unagolla and S.U. Adikary* \\ Department of Materials Science and Engineering \\ University of Moratuwa \\ Sri Lanka
}

\begin{abstract}
Chitosan, a natural biopolymer synthesized from crustaceans shell, plays an influential role in accumulation of heavy metals from wastewater effluents because of its active functional groups. In this study, adsorption characteristics of cadmium and lead were studied using chitosan which was synthesized from locally available shrimps. Kinetic studies were conducted for batch systems using different $\mathrm{pH}$ values of initial metal ion solution and two different degree of deacetylation (DD) values of chitosan. Simplified models such as pseudo-first-order, pseudo-second-order, and intra-particle diffusion equations were used to determine the rate controlling step. A strong dependence of the adsorption capacity on $\mathrm{pH}$ and $D D$ was observed, the capacity increases as $p H$ and $D D$ values increase.
\end{abstract}

Keywords: Adsorption, chitosan, degree of deacetylation, kinetic models

\section{INTRODUCTION}

Heavy metal pollution is a significant problem to the aquatic ecosystems since these heavy metals are potentially toxic even at very trace amounts. Heavy metals are more perilous because they tend to bioaccumilate. (Pathiratne and Senarathna, 2007). Due to these reasons, there is a great interest in removal of heavy metals from waste water effluents. Chitosan is the soluble derivative of chitin, a linear polysaccharide, composed of glucosamine and $\mathrm{N}$ acetyl glucosamine units linked by $\beta(1-4)$ glycosidic bonds. Since the amino groups on the chain are involved in special interaction with metals, chitosan shows good complexation (chelating) ability with metals (Rinaudo, 2006). Degree of deacetylation is an important parameter in chitosan because it directly measures the number of available amine groups in molecular structure of chitosan. Initial $\mathrm{pH}$ of the solution, physiochemical conditions, particle size, processing conditions, degree of deacetylation (DD) and agitation rate are profoundly affected to the above processes. The objective of the present study was to investigate the possible use of chitosan as an alternative adsorption material for removal of cadmium (II) and lead (II) from waste water.

Corresponding author: suadi@uom.lk 


\section{METHODOLOGY}

\section{Isolation of chitosan from shrimp shells}

Shrimp shells were allowed to decay with the $0.05 \mathrm{M}$ acetic acid at room temperature for 8 hours before subjected to chitin processing (Toan et al., 2006). Preconditioned shrimp shells were demineralized with $0.68 \mathrm{M}$ and $1 \mathrm{M} \mathrm{HCl}(1: 10 \mathrm{w} / \mathrm{v})$ at ambient temperature $\left(30^{\circ} \mathrm{C}\right)$ for 6 hours. The residue was washed to maintain neutralized $\mathrm{pH}$ value. It was then dewatered and deproteinized with $0.62 \mathrm{M} \mathrm{NaOH}$ solution $(1: 10 \mathrm{w} / \mathrm{v})$ at ambient temperature $\left(30^{\circ} \mathrm{C}\right)$ for 16 hours. The residue was washed to maintain neutralized $\mathrm{PH}$ value and then it was dewatered. Synthesized chitin was ground and sieved with 250 mesh size. The chitin obtained from the above process was deacetylated in $\mathrm{NaOH}(1: 10 \mathrm{w} / \mathrm{v})$. After deacetylation, the chitosan powder was washed until neutralize the PH (6.5 -7.5) (Adikary et al., 2011; Toan et al., 2006)

\section{Characterization techniques for chitosan}

Degree of deacetylation (DD) is the amount of amino groups formed during the deacetylation process. DD of the chitosan samples were determined by using Fourier transform infrared spectroscopic (FTIR) method and acid base titration method. Chitosan powder $(0.3 \mathrm{~g})$ was dissolved in $30 \mathrm{ml}$ of HCL solution $(0.1 \mathrm{M})$ and mixture was titrated with $\mathrm{NaOH}(0.1 \mathrm{M})$ using methyl orange and aniline blue as indicators until end point (purple to blue green).

\section{Evaluation of experimental data}

Batch experiments were performed for two different DD values of chitosan and three different initial $\mathrm{pH}$ values of the $\mathrm{Pb}$ and $\mathrm{Cd}$ ion solutions. Initial heavy metal ion concentration for both metal ions was taken as $50 \mathrm{mg} / \mathrm{L}(\mathrm{ppm})$. GBC 932 PLUS Atomic Absorption Spectrophotometer was used to find the remaining heavy metal concentration in the solution. Experiments were carried out at room temperature and $450 \mathrm{rpm}$ agitation rate. The adsorption capacity, $\mathrm{q}_{\mathrm{e}}$, was calculated from the difference between the initial single metal concentrations in aqueous solutions, $\mathrm{C}_{\mathrm{o}}$, and the equilibrium concentration $\mathrm{C}_{\mathrm{eq}}$, according to Equation 1.

$q_{\varepsilon}=\frac{\left(c_{s}-c_{e q}\right) W}{W}$

Where,

$\mathrm{V}$ - Volume of aqueous solution in liter (L)

W - Mass of chitosan in grams (g) (Azouaou et al., 2010; Perez-Marin et al., 2007).

To identify the rate-controlling mechanisms during the adsorption of lead and cadmium, three steps were considered:

- Mass transfer of the metallic ion from the bulk solution to the chitosan surface

- Adsorption of the metallic ion into sites

- Internal diffusion of the metallic ion into chitosan

For those purposes, simplified models were applied to evaluate the experimental batch data. 
Pseudo first order kinetics, pseudo second order kinetics and intra-particle diffusion models were used to find the rate controlling mechanism. Pseudo first order model was valid only for a very limited time of the adsorption process (initial 15 minutes) and therefore this model is not suitable to find the rate controlling step (Ahmed, et al., 2005).

Pseudo second order kinetics

Graph was fitted using origin pro 9 analytical software and rate constant and linear regression $\left(\mathrm{R}^{2}\right)$ were calculated according to the equation 2 .

$$
\frac{d q}{d t}=K_{1}\left(q_{e}-q\right)^{2}
$$

Where;

$\mathrm{q}$-Amount of metal absorbed at any time

$\mathrm{q}_{\mathrm{e}}-$ Amount of metal absorbed at equilibrium time

$\mathrm{K}_{1}$ - Pseudo second order rate constant

Intra particle diffusion model

According to the Fickian diffusion low, the amount of adsorption by diffusion controlled dynamics as a function of time can be given by Equation 3 (Hunson and Pitakpoolsil, 2014).

$q=2 C_{0} 5 \sqrt{D t / \pi}$ (3) For analytical convenience, the equation 3 can be rewritten as follows, $q=k_{i} t^{0.5}$

Where;

$\mathrm{K}_{\mathrm{i}}$ - Intraparticle diffusion rate constant

$\mathrm{q}_{\mathrm{t}}$-Amount of metal adsorb at any time

\section{RESULTS AND DISCUSSION}

\section{Characteristics of chitosan}

Increasing of the DD leads to increase the percentage of amine groups is inversely proportional to the percentage of acetyl amide group due to conversion of $-\mathrm{NCHO}$ in to $\mathrm{NH}_{2}$. This structural variation can be clearly observed in the FTIR graphs around $1655 \mathrm{~cm}^{-1}$ since it represent $\mathrm{C}=\mathrm{O}$ stretching of amide bond (Fig. 1). DD obtained from FTIR method varied according to the selected base line system, therefore the titration method is a more reliable method to determine DD (Adikary et al., 2011). 


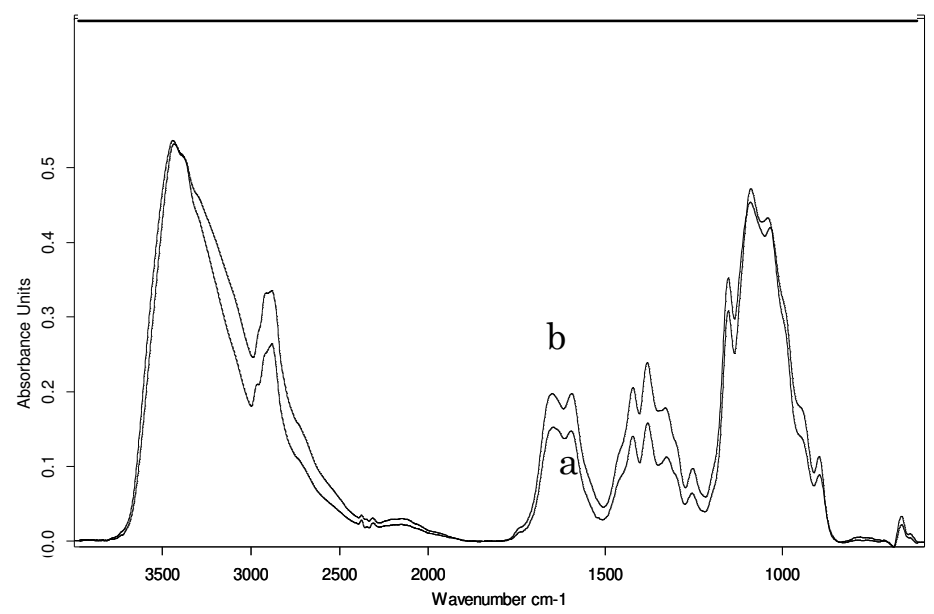

Fig 1. FTIR spectrum of chitosan (a) High DD sample (b) Low DD sample

\section{Adsorption Kinetics}

The adsorption dynamics of $\mathrm{Cd}^{2+}$ and $\mathrm{Pb}^{2+}$ ions were studied using the pseudo second order and intra-particle diffusion model. According to the Fig. 2, the adsorption capacity was significantly affected by the DD of chitosan, due to number of amino groups in chitosan sample and that amino groups are facilitated to adsorb more metal ions from the solution.

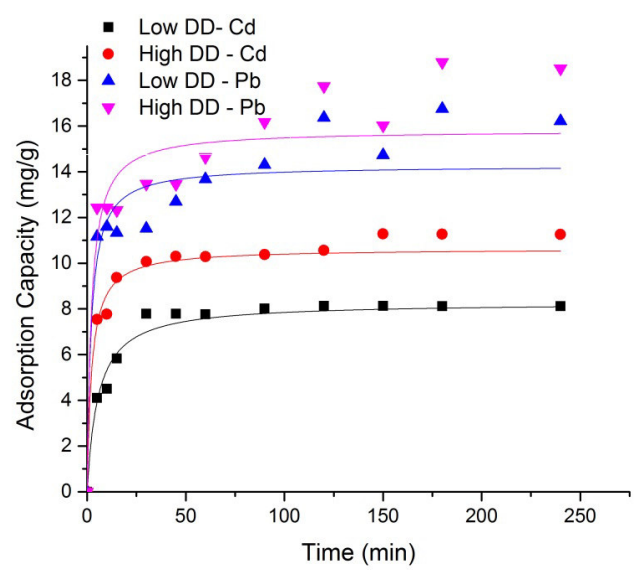

Fig. 2. Pseudo second order model for $\mathrm{Cd}$ and $\mathrm{Pb}$ adsorption at different $\mathrm{DD}$ values of chitosan

According to the Fig. 3, both $\mathrm{Cd}^{2+}$ and $\mathrm{Pb}^{2+}$ indicate a higher adsorption capacity at high initial $\mathrm{pH}$. The tested $\mathrm{pH}$ values for $\mathrm{Cd}^{2+}$ were 3.5, 5.5 and 6.5 and for $\mathrm{Pb}^{2+}$ were $2.0,3.0$ and 4.5. For $\mathrm{Cd}^{2+}$, basic solution $(\mathrm{pH}>7)$ were avoided for the test due to the formation of $\mathrm{Cd}(\mathrm{OH})_{2}$ precipitate. When $\mathrm{pH}$ value is greater than 7 , more cadmium complexes are present in the solution. Likewise, $\mathrm{pH}$ values greater than 5 were avoided for lead because of $\mathrm{Pb}(\mathrm{OH})_{2}$ and at $\mathrm{pH}$ lower than 5, only $\mathrm{Pb}^{2+}$ ions are dominant (Kelesoglu, 2007). The low adsorption 
capacity in acidic solution can be explained by the competition between protons and metallic ions for available amino adsorption sites, and by electrostatic repulsion. The following reaction shows the influence of $\mathrm{pH}$ on the uptake of metallic ions;

Chito $-\mathrm{NH}_{3}{ }^{+}+\mathrm{M}^{2+} \leftrightarrow$ Chito $-\mathrm{NH}_{2}-\mathrm{M}^{2+}+\mathrm{H}^{4}$

The backward reaction of Equation 5 is feasible at higher $\mathrm{H}^{+}$ion concentrations (lower $\mathrm{pH}$ ), reducing the number of binding sites for metallic ions. In addition, the protonation of amino groups in acidic solutions induces an electrostatic repulsion of metal cations that reduces the number of binding sites available for metallic ions. On the other hand, at higher $\mathrm{pH}$, amount of $\mathrm{H}^{+}$ions are reduced and forward reaction is feasible and both competition for binding sites and electrostatic repulsion are reduced. Therefore adsorption performance is improved.

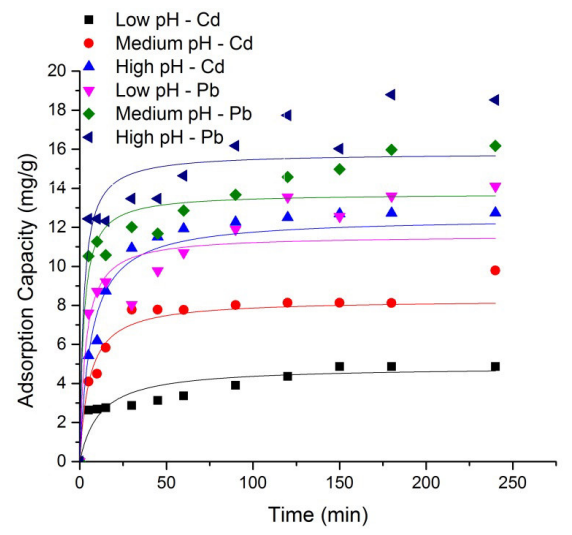

Fig. 3. Pseudo second order model for $\mathrm{Cd}$ and $\mathrm{Pb}$ adsorption at different $\mathrm{pH}$ values of solution

As shown in above figures (Fig. 2 and Fig. 3), pseudo second order model best fit with the experimental data and therefore pseudo second order mechanism is predominant and the overall rate of $\mathrm{Cd}^{2+}$ and $\mathrm{Pb}^{2+}$ adsorption is controlled by adsorption reactions and not by mass transfer. The kinetic constant and linear regression $\left(\mathrm{R}^{2}\right)$ are given in the Table 1 .

Table 1. Kinetic constsnt for lead and cadmium adsorption at different DD and different pH

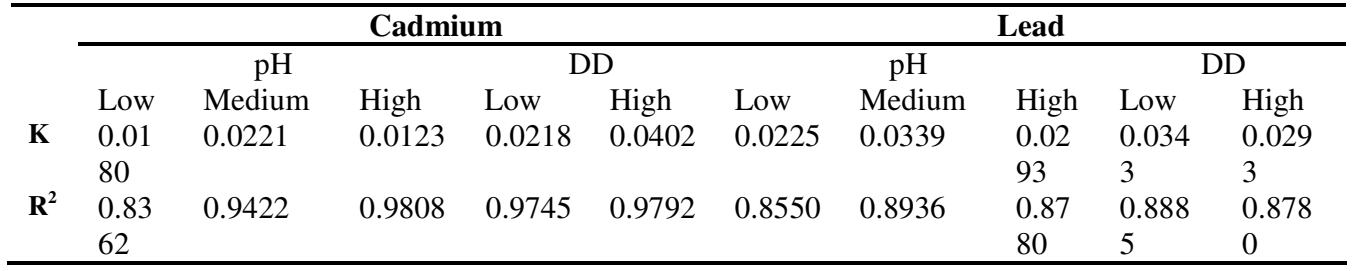


According to the Fig. 4 and Fig. 5, multilinearity can be observed. Initial curved portion of the plot indicates the boundary layer effect while the other linear portion is due to the intraparticle or pore diffusion. The two phases in the plot suggest that the adsorption process facilitates by the surface adsorption and the intra-particle diffusion.

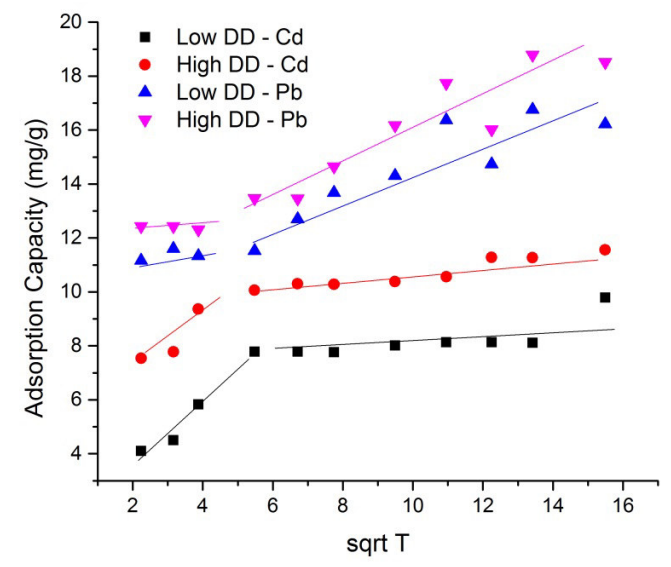

Fig. 4. Intra-particle diffusion model for $\mathrm{Cd}$ and $\mathrm{Pb}$ at different $\mathrm{DD}$ values

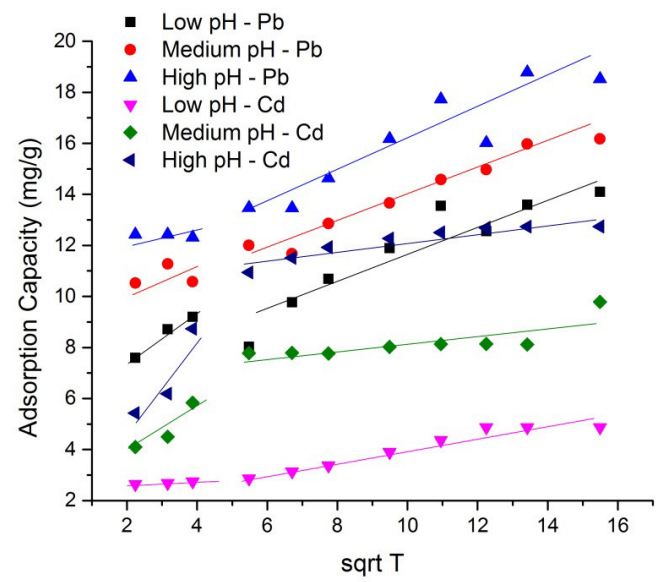

Fig. 5. Intra-particle diffusion model for $\mathrm{Cd}$ and $\mathrm{Pb}$ at different $\mathrm{pH}$ of solution

\section{CONCLUSION}

Chitosan was successfully synthesized from shrimp shells available in Sri Lanka. FTIR spectrum of chitosan showed all the characteristics bond energies of standard chitosan sample. Adsorption rate depend on the $\mathrm{DD}$ value and initial $\mathrm{pH}$ value of the solution. Significantly high rate of adsorption was observed in the chitosan which has higher DD value. According to the results, high $\mathrm{pH}$ values are preferred for good adsorption. Kinetic models were fitted to the experimental results and from that it can be concluded that the adsorption of cadmium and lead heavy metals were controlled by the adsorption reaction and 
not by the mass transfer. According to the study, chitosan could be a good candidate to remove heavy metals from wastewater.

\section{ACKNOWLEDGEMENT}

The authors would like to express their thanks to the Senate Research Committee (SRC) Grant (grant no.SRC/LT/2012/12) of University of Moratuwa for financial support.

\section{REFERENCES}

Adikary, S.U., Samarasekara, A.M.P.B., Unagolla, J.M., Wijesinghe, W.A.R.P. (2011). Effect of Deacetylation Conditions on Synthesis of Chitosan using Shrimp type "penaeus monodon". ERU Proceedings of the $17^{\text {th }}$ annual research symposium, pp 89-91.

Ahmed, A.L., Hameed, B.H. and Sumathi, S. (2005). Adsorption of residue oil from palm oil mill effluent using powder and flake chitosan: Equilibrium and kinetic studies. Water Research, 39, 2483 - 2494.

Azouaou, N., Djaafri, A., Mokaddem, H., and Sadaoui, Z. (2010). Adsorption of cadmium from aqueous solution onto untreated coffee grounds: Equilibrium, kinetics and thermodynamics. Journal of Hazardous Materials, 184, 126 - 134.

Hunson, M. and Pitakpoolsil, W. (2014). Treatment of biodiesel wastewater by adsorption with commercial chitosan flakes: Parameter optimization and process kinetics. Journal of Environmental Management, 133, 284 - 292.

Kelesoglu, S. (2007). Comparative adsorption studies of heavy metal ions on chitin and chitosan biopolymers, M.Sc. thesis, Graduate School of Engineering and Science, Izmir Institute of Technology, Turkey.

Pathiratne, K.A.S. and Senarathna, P. (2007). Accumulation of Heavy metals in a food fish, Mystusgulin inhabiting Bolgoda lake, Sri Lanka. Sri Lanka Journal of Aquatic Science, 12, $61-75$.

Perez-Marin, A.B., Zapata, V.M., Ortuno, J.F., Aguilar, M., Sarez, J. and Llorens, M. (2007). Removal of cadmium from aqueous solutions by adsorption onto orange waste. Journal of Hazardous Materials, B 139, 122 - 131.

Rinaudo, M. (2006). Chitin and chitosan: Properties and applications. Progress in Polymer Science, 31, 603 - 632.

Toan N.V., How Ng C., Aye K.N., Trang T.S. and Stevens W.F. (2006). Production of high quality chitin and chitosan from preconditioned shrimp shells. Journal of Chemical Technology and Biotechnology, 1113 - 1118. 